Patients were $13 \%$ older than controls $(p<0.01)$. Subjects from all groups of less than 70 years had significantly increased PR shortening (mean difference $-9.1 \mathrm{~ms}, \mathrm{p}=0.02$ ) and change in HR (mean difference $15.7 \mathrm{~ms}, \mathrm{p}<0.001$ ) between rest and peak than those over 70 years. Patients had significantly higher Body Mass Indices (BMI) than hypertensive or healthy subjects $(\mathrm{H}=-3.6, \mathrm{p}=0.02, \mathrm{H}=-3.5, \mathrm{p}=0.003$ respectively), as did breathless controls $(\mathrm{H}=3.1, \mathrm{p}=0.012$, $\mathrm{H}=2.8, \mathrm{p}=0.027$ ).

Conclusions Patients with HFpEF have chronotropic incompetence and impaired HR recovery, but there was no evidence of dromotropic incompetence in this study.

\section{OUTCOMES OF RECIPIENTS WITH NON-ISCHAEMIC HEART DISEASE AFTER HEART TRANSPLANTATION}

Maria Simonenko*, Yulia Sazonova, Petr Fedotov, Vadim Rubinchik, Tatjana Pervunina, Aelita Berezina, Kirill Malikov, Andrey Bautin, Vladimir Krasnov, Vladimir Privorotskii, Dmitry Zverev, Lubov Mitrofanova, Maria Sitnikova, German Nikolaev, Michail Gordeev, Michail Karpenko. Federal Almazov North-West Medical Research Centre

\subsection{6/heartjnl-2017-311726.17}

Objective The aim of study was to estimate outcomes of recipients with non-ischaemic chronic heart failure (CHF) after heart transplantation (HT).

Methods From 2010 to 2016 we heart transplanted 80 recipients: $52.5 \%(\mathrm{n}=42)$ - with non-ischaemic CHF (mean age $38.3 \pm 2,2$ years, $\mathrm{n}=4$ children (mean age $-13.5 \pm 2.6$ years)), $47.5 \%(\mathrm{n}=38)$ - with ischaemic heart disease (IHD). Causes of CHF (mean LVEF - 20.9 $1.8 \%$ ) in non-ischaemic group: dilated cardiomyopathy $(61.9 \%, \mathrm{n}=26)$, non-compacted myocardium $(21.4 \%, n=9)$, rheumatic heart disease $(4.8 \%, n=2)$, arrhythmogenic right ventricular dysplasia $(7.1 \%, \mathrm{n}=3)$ and others ( $n=1$ cardiac amyloidosis, $n=1$ cardiac sarcoidosis). Furthermore, 19\% $(\mathrm{n}=8)$ of them were supported by biventricular assist device Berlin Heart EXCOR (duration on support $234 \pm 80$ days) prior HT. According to right heart catheterization results: pulmonary arterial systolic pressure (PASP) 39.9 $\pm 1.9 \mathrm{mmHg}$, pulmonary vascular resistance (PVR) -2.9 \pm 0.2 W.U. Pulmonary hypertension was diagnosed in $66.7 \%$ $(n=28)$ recipients. There was no difference in how much time patients spent in HT waiting list $(158.9 \pm 23.8$ days vs. 152.4 \pm 29.4 days, $p>0.05$ ). All recipients treated by triple-drug therapy (steroids, calcineurin inhibitors, mycophenolic acid), induction: basiliximab - 62\% ( $\mathrm{n}=26)$, thymoglobulin - $38 \%$ $(n=16)$. Outcomes of HT were estimated by survival, TTE results, frequency of rejection, frequency of cardiac allograft vasculopathy (CAV) and cardiopulmonary exercise test results.

Results After 6 year follow-up survival was comparable with IHD recipients (30.4 $\pm 3,1$ months vs. $27.3 \pm 4.2$ months, $\mathrm{p}>0.05$ ), but mortality during 1 st $\mathrm{yr}$ after HT was higher in IHD group $(7.1 \%$ vs. $13.1 \%, \mathrm{p}<0.001)$. After HT non-ischaemic patients spent less time in ICU $(9.3 \pm 0.9$ days vs. 11.5 \pm 1.6 days, $\mathrm{p}>0.05)$, the same as required inotrope support $(6.1 \pm 0.6$ days vs. $8.5 \pm 1.4$ days, $\mathrm{p}<0.05)$. Only 9.5\% $(\mathrm{n}=4)$ of recipients had severe right heart failure and required extracorporeal membrane oxygenation (ECMO) support, 7\% $(n=3)$ - underwent tricuspid valve repair due to severe tricuspid regurgitation. Levosimendan treatment was successfully used in $33.3 \%(n=14)$ patients. In 1 case thoracoscopic pericardial fenestration performed. In 6 months after HT TTE results got to normal values: LVEF - 60.6 $\pm 0.9 \%$, PASP $31.1 \pm 1.3 \mathrm{~mm} \mathrm{Hg}$; results were stable even in 1 year. During 1st yr rejection was found: $\mathrm{R} 2 / 3 \mathrm{~A}-9.5 \%(\mathrm{n}=32)$, AMR1 $2.1 \%(n=7)$. Also in 1 year after HT recipients from nonischaemic group significantly improved their physical capacity $\left(\mathrm{V0}_{2 \text { peak }}-11.7 \pm 0.4 \mathrm{ml} / \mathrm{min} / \mathrm{kg} \quad\right.$ vs. $\quad 17.8 \pm 0.6 \mathrm{ml} / \mathrm{min} / \mathrm{kg}$, $\mathrm{p}<0.001)$. CAV was diagnosed in more than 1 year of followup. Furthermore, frequency of CAV was more often in IHD group $(9.5 \%(n=4)$ vs. $13.2 \%(n=5), p<0.05)$.

Conclusion After HT survival and complications are comparable in recipients with IHD and non-ischaemic group.

\section{UPGRADE TO DEFIBRILLATOR IN PATIENTS RECEIVING CARDIAC RESYNCHRONISATION THERAPY PACEMAKERS}

${ }^{1}$ Shveta Monga*, 'Dominic Haigh, 'David Royan, ${ }^{1}$ Paul Foley, ${ }^{1}$ Badrinath Chandrasekaran. ${ }^{1}$ Great Western hospital NHS trust

\subsection{6/heartjnl-2017-311726.18}

Introduction In patients with advanced cardiac failure, the choice between CRT with (CRT-D) or without a defibrillator (CRT-P), remains a contentious issue. There is insufficient evidence from randomised controlled trials and guidelines do not make any firm recommendations. This leaves room for physician discretion and has led to wide variation in worldwide practice.

Study aim and basic methods The focus of our study was to evaluate the characteristics of CRT-P patients in the real world setting and analyse how many patients with CRT-P implant required an upgrade to CRT-D in the follow-up period.

This was a retrospective single centre observational study looking at 122 patients implanted with CRT from 2011 to 2015. Data was collected from patient electronic records and follow-up visits. The median follow-up duration was 2.2 (1.23.3) years. Data was analysed using Microsoft excel and SPSS. Results 1. The patients implanted with CRT-P were elderly, had more advanced heart failure with multiple co-morbidities and mostly females.

2. Only 3 out of 122 patients (2.5\%) were upgraded from CRT-P to CRT-D. All these patients were males, above 75 years of age with a primary prevention indication for CRT-D and had upgrade following episodes of symptomatic ventricular tachycardia (Figure 1).

\begin{tabular}{|l|l|l|}
\hline & Upgrade & CRT-P \\
\hline Number of patients & 3 & 122 \\
\hline Mean Age in yrs & 77.3 & 79.2 \\
\hline Female & None & $24 \%$ \\
\hline Ischaemic heart disease & $33 \%$ & $48 \%$ \\
\hline Dilated cardiomyopathy & $67 \%$ & $51 \%$ \\
\hline
\end{tabular}

Abstract 18 Figure 1 Clinical profile of upgraded patients compared to rest of the CRT-P population

3. The main cause of mortality in patients implanted with CRT-P was non-cardiac vs. cardiac $(5.7 \%$ vs. $15.6 \%$, p value $<0.001)$. Majority of deaths in the cardiac group were due to pump failure. Only $10 \%$ of patients in this group had documented non sustained ventricular arrhythmias.

Conclusion In our study population, the number of CRT-P patients who needed upgrade with additional defibrillator was 


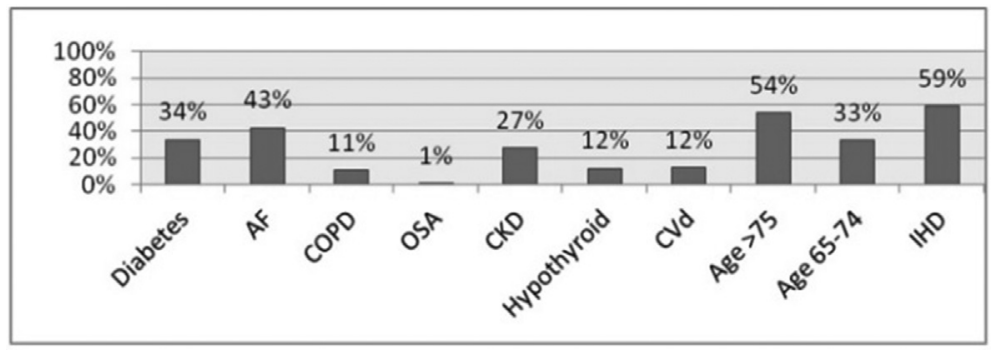

Abstract 19 Figure 1 Modified Frailty Index Clinical parameters and prevalence in our study population (Potential for a maximum and minimum modified frailty index of 11 and 0 )

surprisingly small in medium term follow up. This implies that clinician guided selection criteria is an appropriate method of selecting patients who may not derive additional benefit from a defibrillator.

\section{MODIFIED FRAILITY INDEX AS PREDICTOR OF OUTCOME FOR PATIENTS IMPLANTED WITH CARDIAC RESYNCHRONISATION THERAPY}

"Shveta Monga, Dominic Haigh, David Royan, Chitsa Seyani, Richard Francis, Paul Foley, Badrinath Chandrasekaran. 'Great Western hospital NHS trust

\subsection{6/heartjnl-2017-311726.19}

Introduction Frailty is a multidimensional syndrome, contributing significantly to the morbidity and mortality of elderly patients. With an increase in average life expectancy, there are a growing number of frail individuals with multiple co-morbidities and cardiac failure. Although multiple studies have shown that in selected patients cardiac resynchronisation therapy (CRT) improves morbidity and mortality, the role of frailty has not been rigorously evaluated in these individuals. There are no current risk scores for frailty to help refine the predicted impact of CRT on survival in elderly patients.

Study aim and basic methods The focus of our study was to develop and review the role of an easy-to -use frailty index as a predictor of outcome in patients being considered for CRT and suggest ways in which frailty assessment could be incorporated into clinical practice.

This was a retrospective study of 265 patients implanted with CRT from 2011 to 2015. Data was collected from

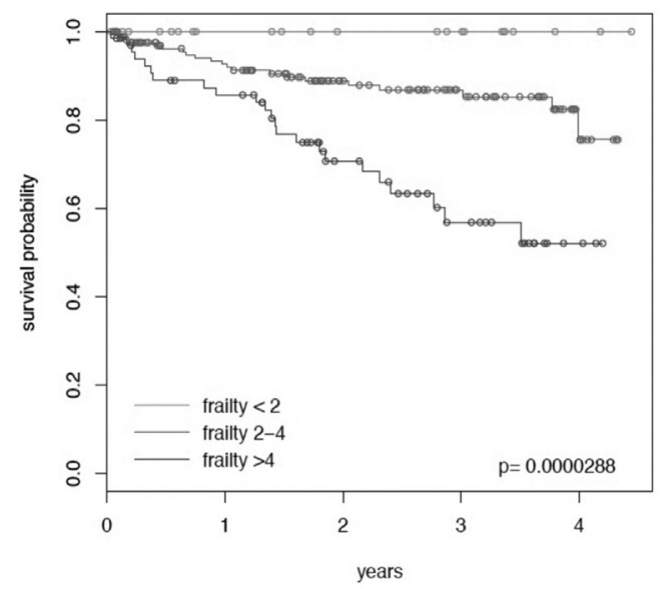

patient electronic records and follow-up visits. The median follow-up duration was $2.2(1.2-3.3)$ years.

A modified frailty index was formulated using the Canadian Study of Health and Ageing. The modified frailty index was based on 9 of the potential 70 Canadian Study of Health and Ageing clinical deficits. These variables were chosen based on the likelihood of being recorded in the electronic records bearing in mind the practical future application of the frailty index in a clinical setting. Each deficit was given 1 point except age $>75$ years (given 2 points). The clinical parameters used and their prevalence in our study population is shown in figure 1 .

Data were analysed using SPSS and Microsoft excel using Chi square test, survival analysis and ROC analysis.

Results 1. In patients implanted with CRT, mortality was found to be higher in patients with a greater frailty index. ( $\mathrm{p}$ value 0.00003 ) (Image 1)

2. Mean modified frailty index in those who died was higher (4.4; SD 1.5) than in those who lived (3.1; SD 1.5)

3 . In ROC analysis, frailty index $>2$ was a significant predictor of mortality in our subgroup of patients (sensitivity 97.7\%, AUC 0.73, 95\% CI=0.14-0.32, p<0.0001) (Image 2)

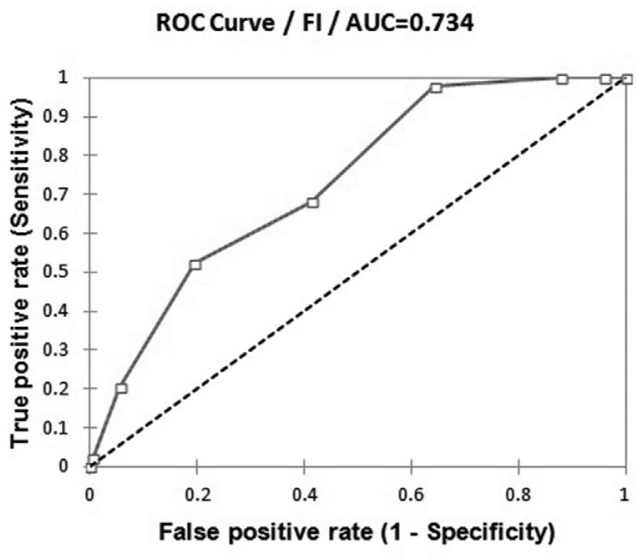

Abstract 19 Figure 3

Conclusion In patients implanted with CRT mortality was found to be significantly greater in more frail patients, represented by a higher modified frailty index. Therefore the modified frailty index may be a useful tool in predicting mortality, helping to guide patient and family expectations when considering device therapy. 